\title{
Regional Anesthesia for Abdominal Surgery in a Patient with Severe Chronic Respiratory Failure: A Case Report
}

\author{
Misoon Lee, Younghoon Woo, Jaewoong Jung, Yang-Hoon Chung, Bon Sung Koo, Sung-Hwan Cho \\ Department of Anesthesiology and Pain Medicine, Soonchunhyang University Bucheon Hospital, Bucheon, Korea
}

\begin{abstract}
General anesthesia is associated with a risk for postoperative pulmonary complications. The risk is even higher in patients with chronic respiratory failure, and postoperative mortality rates are high. Proper perioperative anesthetic management is important in such patients. Therefore, it is essential to optimize the patient's physical status before anesthesia and to determine the optimal anesthesia technique based on the pre-anesthesia evaluation of the patient's pulmonary function. We successfully performed abdominal surgery under spinal anesthesia in a patient with severe chronic respiratory failure.
\end{abstract}

Keywords: Regional anesthesia; Chronic obstructive pulmonary disease; Respiratory insufficiency; Postoperative complications; Lung; Pulmonary tuberculosis; Case report

\section{INTRODUCTION}

Patients with pre-existing respiratory diseases are at a greater risk for pulmonary complications, such as respiratory infections, respiratory failure, atelectasis, pneumothorax, bronchospasm, and aspiration, following general anesthesia [1]. Abdominal surgery significantly increases the risk for respiratory complications [2]. Various techniques can be used to minimize the risk for postoperative pulmonary complications in patients with pre-existing respiratory failure. Regional anesthesia is a useful technique to optimize the patient's physical status and improve the prognosis. Here, we report the use of spinal anesthesia in a patient with respiratory failure who underwent lower abdominal surgery.

\section{CASE REPORT}

A 67-year-old female was scheduled to undergo total abdominal hysterectomy and bilateral salpingectomy. She had a history of hypertension, diabetes mellitus, cerebral infarction, asthma, chronic obstructive pulmonary disease (COPD), and tuberculosis-destroyed lung due to pulmonary tuberculosis 30 years prior. The patient was $161 \mathrm{~cm}$ tall, weighed $40 \mathrm{~kg}$, and American Society of
Anesthesiologists status IV. A chest radiograph showed that the left lung cavity was destroyed and revealed tuberculosis sequelae in the upper right lung (Fig. 1). Preoperative pulmonary function test showed that the forced expiratory volume in 1 second (FEV1) and the forced vital capacity (FVC) were $0.34 \mathrm{~L}(18 \%)$ and $1.43 \mathrm{~L}$ (55\%), respectively. The FEV1/FVC ratio was $24 \%$ (Table 1 ). The arterial blood gas analysis showed a $\mathrm{pH}$ of $7.33, \mathrm{PaCO}_{2}$ of $57 \mathrm{~mm} \mathrm{Hg}$, $\mathrm{PO}_{2}$ of $162 \mathrm{~mm} \mathrm{Hg}$, and $\mathrm{HCO}^{3-}$ of $30.1 \mathrm{mmol} / \mathrm{L}$ (Table 2). Neuraxial anesthesia was chosen because of the severely compromised respiratory function. The patient was taking aspirin and clopidogrel due to a history of cerebral infarction. Clopidogrel was discontinued for more than 5 days to reduce the risk of bleeding.

Standard monitoring, including three-lead electrocardiography, pulse oximetry, and noninvasive blood pressure monitoring, was performed in the operation room and the left radial artery was cannulated for continuous blood pressure monitoring. The patient was positioned laterally for combined spinal-epidural anesthesia. A midline approach to L4-5 using a 25G Quinke needle for dural puncture was followed by the injection of $12 \mathrm{mg} 0.5 \%$ hyperbaric bupivacaine. The L2-3 interspace was identified by palpation and an epidural catheter was inserted. Anesthesia up to the T6 dermatome was confirmed using the cold-sensation test 5 minutes after
Correspondence to: Misoon Lee

Department of Anesthesiology and Pain Medicine, Soonchunhyang University Bucheon Hospital, 170 Jomaru-ro, Wonmi-gu, Bucheon 14584, Korea

Tel: +82-32-621-5339, Fax: +82-32-621-5322, E-mail: misoonlnee@schmc.ac.kr

Received: Nov. 25, 2021 / Accepted after revision: Dec. 9, 2021
(C) 2021 Soonchunhyang Medical Research Institute This is an Open Access article distributed under the terms of the Creative Commons Attribution Non-Commercial License (https://creativecommons.org/licenses/by-nc/4.0/). 


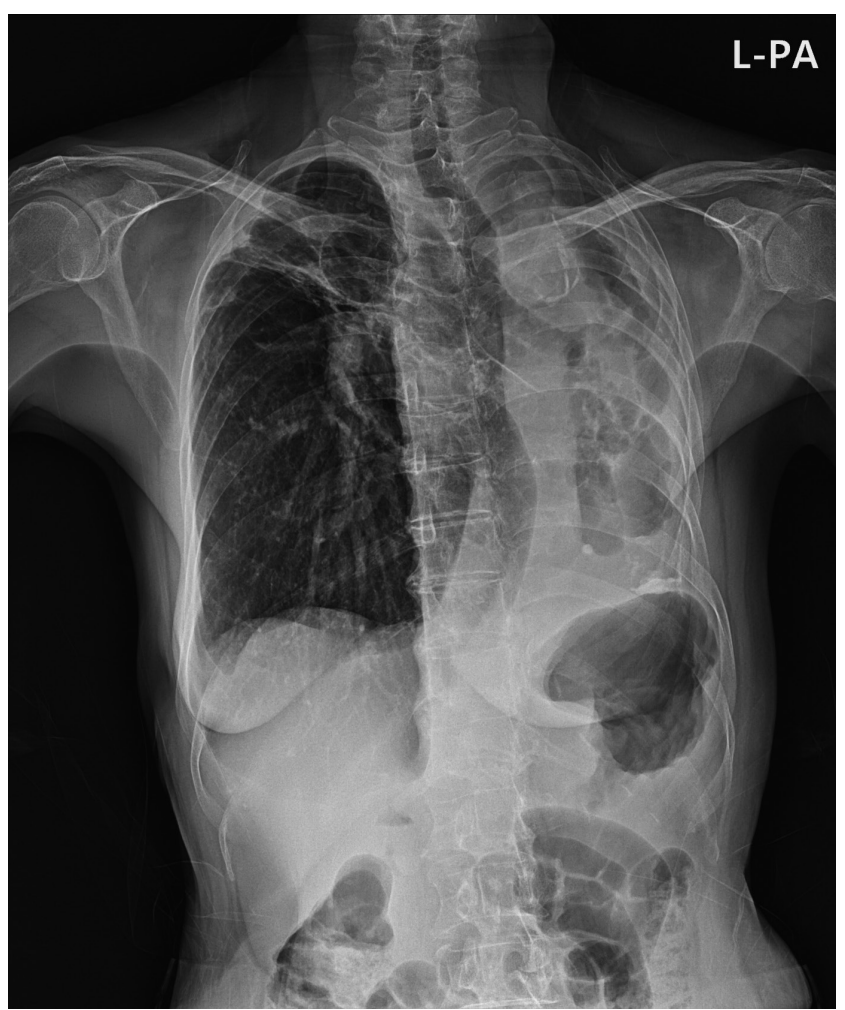

Fig. 1. Preoperative chest radiography. L-PA, left posteroanterior.

the intrathecal injection. Dexmedetomidine was administered intravenously for sedation, and $4 \mathrm{~L} / \mathrm{min}$ oxygen was administered using a nasal cannula. Vital signs, including oxygen saturation, remained stable intraoperatively. The operation was completed in 1 hour and 2 minutes without the need for additional local-anesthetic administration. There were no intraoperative complications, and the patient was transferred to the post-anesthetic care unit.

This study was approved by the institutional review board of Soonchunhyang University Bucheon Hospital (approval no., SCHBC 2021-03-029). The board waived the requirement for patients' informed consent because this study was a retrospective medical record review.

\section{DISCUSSION}

Patients with respiratory failure are more likely to develop perioperative pulmonary complications following general anesthesia. COPD patients are at a higher risk for postoperative mortality [3]. Abdominal surgery is associated with a higher risk for postoperative pulmonary complications compared to other surgical procedures. Therefore, sufficient pre-anesthesia evaluation, with special
Table 1. Preoperative pulmonary function test

\begin{tabular}{lccc}
\hline Variable & Predicted value & Actual value & Pre \%Ref \\
\hline FVC (L) & 2.60 & 1.43 & 55 \\
FEV1 (L) & 1.86 & 0.34 & 18 \\
FEV1/FVC (\%) & 72.00 & 24.00 & \\
FEF 25\%-75\% (L/sec) & 2.23 & 0.22 & 10 \\
PEF (L/sec) & 5.23 & 0.88 & 17 \\
\hline
\end{tabular}

FVC, forced vital capacity; FEV1, forced expiratory volume in one second; FEF $25 \%$ $75 \%, 25 \%-75 \%$ of forced expiratory flow; PEF, peak expiratory flow; Pre \%Ref, ratio of actual value to predicted value.

Table 2. Perioperative arterial blood gas analysis

\begin{tabular}{lcccccc}
\hline Variable & $\mathrm{pH}$ & $\mathrm{PaCO}_{2}(\mathrm{~mm} \mathrm{Hg})$ & $\mathrm{PO}_{2}(\mathrm{~mm} \mathrm{Hg})$ & $\mathrm{BE}(\mathrm{mEq} / \mathrm{L})$ & $\mathrm{SaO}_{2}(\%)$ & $\mathrm{FiO}_{2}$ \\
\hline PreOP & 7.56 & 25 & 163 & 1.5 & 100 & 0.21 \\
IntraOP & 7.42 & 51 & 312 & 7.2 & 100 & 0.36 \\
PostOP & 7.33 & 57 & 162 & 2.8 & 99 & 0.21 \\
\hline
\end{tabular}

$\mathrm{pH}$, arterial $\mathrm{pH} ; \mathrm{PaCO}_{2}$, arterial carbon dioxide tension; $\mathrm{PaO}_{2}$, arterial oxygen tension; $\mathrm{BE}$, arterial base excess; $\mathrm{SaO}_{2}$, arterial oxygen saturation; $\mathrm{FiO}_{2}$, fraction of inspired oxygen; PreOP, a day before operative day; IntraOP, intraoperative period; PostOP, about 8 hours after the end of the operation.

attention to the patient's respiratory function, should precede abdominal surgery in patients with severe respiratory failure. The main consideration for anesthetic management in patients with respiratory failure is the prevention of postoperative pulmonary complications and exacerbation of the underlying diseases. Perioperative optimization of the patient's general condition and thorough pre-anesthesia respiratory function evaluation are crucial. In addition, the optimal anesthetic technique must be selected to minimize the effects of anesthesia on respiratory function.

Our patient had severely compromised pulmonary function due to pre-existing COPD, asthma, and lung destruction. Combined spinal-epidural anesthesia was chosen for the patient considering her pulmonary function. As the patient was scheduled for lower abdominal surgery with the estimated operation time of about an hour, spinal anesthesia was sufficient for the procedure.

Regional anesthesia has fewer effects on the respiratory system than general anesthesia. General anesthesia requires tracheal intubation, mechanical ventilation, and administration of neuromuscular blockers, which may lead to bronchospasm, ventilation perfusion mismatch, lung infiltration, atelectasis, and decreased respiratory-muscle function due to residual muscle relaxation. The use of regional anesthesia is sufficient for abdominal surgeries without the risk for these complications $[4,5]$. Regional anesthesia significantly reduces the incidence of pulmonary complications in 
COPD patients during major abdominal surgeries [6]. However, regional anesthesia does have some effects on respiratory function and pulmonary complications have been reported. Several studies have reported bronchospasm in asthmatic patients following epidural and spinal anesthesia $[7,8]$. The sympathetic block that controls the bronchi results in relative dissonance of the autonomic nervous system, which may cause bronchospasm. In addition, relaxation of the respiratory muscles due to upper thoracic spinal block may exacerbate hypercapnia and dyspnea [9]. However, despite the potential risks, regional anesthesia has significant advantages in patients with compromised pulmonary function.

In conclusion, compared to general anesthesia, neuraxial anesthesia is associated with significantly reduced risk for postoperative pulmonary complications during abdominal surgery in patients with respiratory failure.

\section{CONFLICT OF INTEREST}

No potential conflict of interest relevant to this article was reported.

\section{REFERENCES}

1. Miskovic A, Lumb AB. Postoperative pulmonary complications. Br J Anaesth 2017;118:317-34.

2. Brooks-Brunn JA. Predictors of postoperative pulmonary complications following abdominal surgery Chest 1997;111:564-71.

3. Mallon JS, Edelist G. Risk factors of importance-the patient. Probl Anesth 1992;6:193-204.

4. Guay J, Choi PT, Suresh S, Albert N, Kopp S, Pace NL. Neuraxial anesthesia for the prevention of postoperative mortality and major morbidity: an overview of Cochrane systematic reviews. Anesth Analg 2014;119: 716-25

5. Savas JF, Litwack R, Davis K, Miller TA. Regional anesthesia as an alternative to general anesthesia for abdominal surgery in patients with severe pulmonary impairment. Am J Surg 2004;188:603-5.

6. van Lier F, van der Geest PJ, Hoeks SE, van Gestel YR, Hol JW, Sin DD, et al. Epidural analgesia is associated with improved health outcomes of surgical patients with chronic obstructive pulmonary disease. Anesthesiology 2011;115:315-21.

7. Wang CY, Ong GS. Severe bronchospasm during epidural anaesthesia. Anaesthesia 1993;48:514-5.

8. Rodilla-Fiz AM, Gomez-Garrido M, Martinez-Lopez F, Monsalve-Naharro JA, Casa GL, Lopez-Perez A. Bronchospasm triggered by spinal anaesthesia: case report and review of the literature. Rev Colomb Anestesiol 2016;44:179-81.

9. Steinbrook RA. Respiratory effects of spinal anesthesia. Int Anesthesiol Clin 1989;27:40-5. 